\title{
Epidemiology and molecular characterization of Neisseria lactamica carried in 11-19 years old students in Salvador, Brazil
}

\author{
Ana Rafaela Silva Simões Moura ${ }^{a}$, Cécilia Batmalle Kretz ${ }^{b}$, Ítalo Eustáquio Ferreira ${ }^{a}$, \\ Amélia Maria Pithon Borges Nunes ${ }^{\mathrm{a}}$, Ivano de Filippis ${ }^{\mathrm{c}}$, José Cássio de Moraes ${ }^{\mathrm{d}}$, \\ Mitermayer Galvão Reis ${ }^{\mathrm{a}}$, Alan John Alexander McBride ${ }^{\mathrm{a}, \mathrm{e}}$, Xin Wang ${ }^{\mathrm{b}}$, Leila Carvalho Campos ${ }^{\mathrm{a}, *}$ \\ a Laboratório de Patologia e Biologia Molecular, Instituto Gonçalo Moniz, FIOCRUZ-BAHIA, Rua Waldemar Falcão 121, 40296-710, Salvador BA, Brazil \\ ${ }^{\mathrm{b}}$ Meningitis and Vaccine Preventable Diseases Branch, Division of Bacterial Diseases, Centers for Disease Control and Prevention, 1600 Clifton Road NE, Atlanta GA \\ 30333, USA \\ ${ }^{\mathrm{c}}$ Instituto Nacional de Controle de Qualidade em Saúde - INCQS, FIOCRUZ, 21040-900, Rio de Janeiro RJ, Brazil \\ ¿ Faculdade de Ciências Médicas da Santa Casa de São Paulo, 01220200, São Paulo SP, Brazil \\ e Núcleo de Biotecnologia, Centro de Desenvolvimento Tecnológico, Universidade Federal de Pelotas, Campus Universitário s/n, 96160-000, Pelotas RS, Brazil
}

\section{A R T I C L E I N F O}

\section{Keywords:}

Neisseria lactamica

Neisseria meningitidis

Oropharyngeal carriage

Vaccines

Meningococcal disease

Whole genome sequencing

\begin{abstract}
A B S T R A C T
Neisseria lactamica is a nonpathogenic commensal bacterium that is potentially associated with the development of natural immunity against $N$. meningitidis. However, the genetic variation present in natural populations of $N$. lactamica has not been fully investigated. To better understand its epidemiology and genetic variation, we studied $N$. lactamica carriage in 1200 students aged 11-19 years old in Salvador, Brazil. The carriage prevalence was $4.5 \%$ (54/1200), with no statistical difference among sex and age, although we observed a trend towards higher carriage prevalence among 11-year-old individuals. Whole genome sequence analysis revealed a high genetic diversity among the isolates, with the presence of 32 different STs, 28 (87.5\%) of which were new. A total of $21 / 50(42 \%)$ isolates belonged to three different clonal complexes. While none of the isolates contained nadA or $f H p b$ alleles, we detected 21 FetA variants, 20 NhbA variants and two variants of PorB. The data provide detailed information on circulating $N$. lactamica isolates in adolescents in Brazil and are complementary to studies in other countries.
\end{abstract}

\section{Introduction}

Neisseria lactamica is a lactose fermenting diplococcus closely related to $N$. meningitidis, which lives in a commensal relationship with humans. This bacterium is frequently isolated from the nasopharynx of children, and is rarely associated with invasive disease as it lacks several virulence factors usually found in Neisseria meningitidis (Changal et al., 2016; Everts et al., 2010).

$N$. lactamica is of interest as it has been implicated in the age-related development of natural immunity against $N$. meningitidis (Gold et al., 1978). Although poorly understood, the prevalence of $N$. lactamica carriage in young children $(<5$ years of age) is significantly higher compared to $N$. meningitidis. Furthermore, these children developed significant IgG responses that were cross-reactive with serogroup A, B, and C meningococci soon after colonization with $N$. lactamica (Gold et al., 1978).

$N$. lactamica does not express the meningococcus protective capsule
(Kim et al., 1989) and the outer-membrane protein PorA (Ward et al., 1992). However, there is similar relatedness among some outer membrane proteins, including porin B (PorB) (Bennett et al., 2008), ironregulated enterobactin (FetA) (Bennett et al., 2009) and neisserial heparin-binding antigen (NhbA), although the variants are mainly not overlapping in the two species (Lucidarme et al., 2013).

The evidence of cross-reactivity responses against common antigens (Cann and Rogers, 1989; Troncoso et al., 2002) encouraged the development of anti-meningococcal vaccines based on $N$. lactamica (Griffiss et al., 1991; Finney et al., 2008; Gorringe et al., 2009).

Some of the subcapsular antigens common to $N$. lactamica and $N$. meningitidis are included in the multiple component serogroup B meningococcal vaccine, 4CMenB (Serruto et al., 2012). The use of vaccines containing surface proteins shared with $N$. lactamica could interfere in the colonization of the nasopharynx by $N$. lactamica, potentially hampering the acquisition of natural immunity (Lucidarme et al., 2013; Troncoso et al., 2002). The impact of meningococcal vaccines on

\footnotetext{
* Corresponding author

E-mail address: lccampos@bahia.fiocruz.br (L.C. Campos).
} 
neisserial species with similar surface proteins warrants further investigation (Toneatto et al., 2017).

Although there is a degree of relatedness between some of the surface antigens, the commensal $N$. lactamica has not been prioritized to the same degree as $N$. meningitidis, especially with regard to studies on epidemiology and genetic variation (Alber et al., 2001; Bennett et al., 2005; Kristiansen et al., 2012; Lucidarme et al., 2013). Furthermore, there is no information regarding the circulation and genetic diversity of $N$. lactamica isolates in Brazil.

Previously, we conducted a cross-sectional study to assess the meningococcal carriage status of 11-19-year-old student's resident in Salvador (Nunes et al., 2016; Moura et al., 2017). Although the laboratory methodology was primarily designed for meningococcus isolation, all lactose-fermenting Gram-negative diplococci were registered and stored for further investigation. In the present study, we describe the epidemiology and the genetic profiles of $N$. lactamica isolates recovered from 11 to 19-year-old carriers in Salvador, Brazil.

\section{Material and methods}

\subsection{Ethical considerations}

This study was approved by the Ethics Committee of the Instituto Gonçalo Moniz, FIOCRUZ-BA (CAAE \#16099713.1.0000.0040). Written informed consent from all study participants (or guardians) was obtained before sample and data collection.

\subsection{Isolation and identification of $N$. lactamica}

$N$. lactamica isolates $(n=54)$ were recovered from the oropharyngeal swabs collected from 1200 students, aged 11-19 years old, attending a total of 134 different public schools in Salvador, Brazil, during September to December 2014 (Nunes et al., 2016). The swabs were immediately used to inoculate selective agar medium (modified Thayer-Martin agar containing vancomycin, colistin, nystatin, and trimethoprim) and transferred to a polystyrene tube containing $1 \mathrm{~mL}$ of skim milk-tryptone-glucose-glycerin (STGG) transport medium (O'Brien et al., 2001). After $24-48 \mathrm{~h}$ of incubation, the plates were inspected and colonies with the characteristic morphology of Neisseria spp. were subcultured on blood agar medium for species identification by Gram staining, oxidase reaction, and carbohydrate utilization tests. The results were confirmed using API-NH1 strips (bioMérieux, Hazelwood, MO, USA), as described previously (Nunes et al., 2016). N. lactamica isolates were stored in brain heart infusion (BHI) broth containing $20 \%$ (v/v) glycerol at $-80^{\circ} \mathrm{C}$.

\subsection{Molecular characterization}

Of the $54 \mathrm{~N}$. lactamica isolates, 50 were characterized by whole genome sequencing (WGS). Genomic DNA was extracted as previously described (Kretz et al., 2016), and sequenced using MiSeq v2 chemistry (Illumina, San Diego, CA, USA). Genome assembly was carried out using CLC Genomics Workbench, ver. 9.0.0 (CLC bio, Aaarhus, Denmark) with read trimming and mapping of reads back to contigs. The multilocus sequence typing (MLST) alleles, sequence types (STs) and clonal complexes (cc) were identified by comparison of the assembled genomes with Neisseria PubMLST database (http://pubmlst.org/ neisseria/), using a BLAST search (https://blast.ncbi.nlm.nih.gov/ Blast.cgi). The presence and diversity of porB, fetA and nhba were investigated using sequence analysis as previously described (Kretz et al., 2016). Partial protein sequences were used for PorB, FetA typing, and full-length protein sequences were used for NHBA typing.

\subsection{Phylogenetic analysis}

Single nucleotide polymorphisms (SNPs) were identified using kSNP version 3 software (Gardner and Hall, 2013) with a kmer length of 25. A maximum likelihood phylogenetic tree was constructed from the core SNPs and the Tamura-Nei model, using MEGA7 (Tamura et al., 2013) and 500 bootstraps interactions.

\subsection{Data analysis}

Statistical analysis was done using STATA statistical software version 12 (College Station, TX, USA). The prevalence of $N$. lactamica carriage was calculated for the total sample and for subgroups (sex and age). Univariate analysis to identify exposure associated with $N$. lactamica carriage was performed; the chi-square test was used to determine statistical significance.

\section{Results}

\subsection{N. lactamica carriage}

Among the 1200 students screened, $N$. lactamica was isolated from $54(4.5 \%)$ individuals (Nunes et al., 2016). There was no significant difference in carriage prevalence based on gender: 31 (57.4\%) females and $23(42.6 \%)$ males. Although the $N$. lactamica carriage rate was slightly higher among 11-year-old students (9.7\%), it was not statistically significant (Fig. 1). The prevalence of $N$. lactamica carriage across the various age groups was similar to that of $N$. meningitidis in the same population (Fig. 1). Only one participant was co-colonized by both $N$. meningitidis and $N$. lactamica.

\subsection{Molecular characterization}

A total of $50 \mathrm{~N}$. lactamica isolates identified by conventional methods were characterized by WGS. Thirty-two different STs were identified, $28(87.5 \%)$ of which were new. The majority of the isolates (29/50, 58\%) lacked association within any known cc in the PubMLST database. A total of $21(42 \%)$ isolates belonged to three different cc: cc613 (13/50; 26\%); cc1494 (5/50; 10\%); cc624 (3/50; 6\%). We were unable to determine the ST of one isolate (M37159) due to a deletion of the pdhC housekeeping gene (Table 1). The phylogenetic analysis showed a high level of genetic variability with many different ST identified; and isolates belonging to the same ST and/or cc type to cluster together (Fig. 2). A total of 11329 core SNPs were identified with a difference of 80-5615 SNPs between all isolates analyzed.

Among the outer membrane proteins, two PorB variants were identified: $3-599(10 / 50 ; 20 \%)$ and 3-596 (40/50; 80\%), the latter being novel and most prevalent among the isolates (Table 1). All but nine of the isolates contained the FetA VR (variable region), with 21 FetA VRs in total. The most prevalent was F1-29 (12/41; 29.3\%), and we identified four new variants: F1-143 (2/41; 4.9\%), F1-204 (1/41; 2.4\%), F4-68 (1/41; 2.4\%), and F5-120 (1/41; 2.4\%) (Table 1).

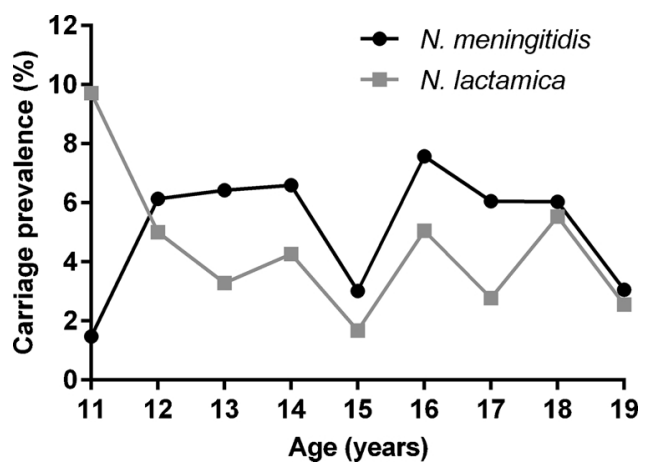

Fig. 1. Carriage prevalence for $N$. lactamica and $N$. meningitidis among adolescents resident in Salvador, Brazil, 2014 by age group. Note, the data for $N$. meningitidis was offset by $0.5 \%$ on the $y$-axis for clarity. 
Table 1

Genetic profile of 50 Neisseria lactamica isolates.

\begin{tabular}{|c|c|c|c|c|c|}
\hline ID N $^{\circ}$ & ST & Clonal Complex & PorB & FetA & NhbA \\
\hline M37058 & 11636 & 1494 & $3-599$ & F1-105 & 291 \\
\hline M37063 & 11637 & NA & $3-596$ & F5-88 & 88 \\
\hline M37071 & 11637 & NA & $3-596$ & F4-17 & 88 \\
\hline M37073 & 11639 & 613 & $3-599$ & $\mathrm{~F} 4-68^{\mathrm{a}}$ & $305^{\mathrm{b}}$ \\
\hline M37078 & 11640 & 624 & $3-596$ & - & $268^{\mathrm{b}}$ \\
\hline M37079 & 11641 & NA & $3-596$ & $\mathrm{~F} 5-120^{\mathrm{a}}$ & $533^{\mathrm{b}}$ \\
\hline M37083 & 11637 & NA & $3-596$ & F5-88 & 88 \\
\hline M37084 & 11457 & 613 & $3-596$ & F1-33 & $305^{\mathrm{b}}$ \\
\hline M37091 & 11643 & NA & $3-596$ & $-{ }^{\top}$ & 526 \\
\hline M37094 & 11644 & NA & $3-596$ & $\mathrm{~F} 1-143^{\mathrm{a}}$ & 928 \\
\hline M37101 & 11645 & NA & $3-596$ & F1-68 & 88 \\
\hline M37105 & 11644 & NA & $3-596$ & $\mathrm{~F} 1-143^{\mathrm{a}}$ & 928 \\
\hline M37107 & 11647 & NA & $3-596$ & F1-120 & 92 \\
\hline M37110 & 11643 & NA & $3-596$ & $-{ }^{\top}$ & 526 \\
\hline M37119 & 11649 & NA & $3-596$ & $\mathrm{~F} 2-7$ & 88 \\
\hline M37123 & 11650 & NA & $3-599$ & $\mathrm{~F} 1-204^{\mathrm{a}}$ & $268^{\mathrm{b}}$ \\
\hline M37130 & 11651 & NA & $3-596$ & F4-19 & 526 \\
\hline M37131 & 11636 & 1494 & $3-599$ & F1-105 & 262 \\
\hline M37132 & 11653 & 613 & $3-596$ & F1-29 & 932 \\
\hline M37136 & 11654 & NA & $3-596$ & F1-29 & 88 \\
\hline M37137 & 11655 & NA & $3-596$ & $-\tau$ & 88 \\
\hline M37140 & 613 & 613 & $3-599$ & F2-7 & $305^{\mathrm{b}}$ \\
\hline M37142 & 11637 & NA & $3-596$ & F4-17 & 88 \\
\hline M37143 & 11654 & NA & $3-599$ & F1-29 & 88 \\
\hline M37146 & 11658 & NA & $3-596$ & F5-13 & $268^{\mathrm{b}}$ \\
\hline M37147 & 11654 & NA & $3-596$ & F2-1 & 88 \\
\hline M37155 & 11660 & NA & $3-596$ & F1-29 & 88 \\
\hline M37157 & 613 & 613 & $3-596$ & F1-29 & $175^{\mathrm{b}}$ \\
\hline M37158 & 624 & 624 & $3-596$ & -9 & 929 \\
\hline M37159" & & & $3-596$ & $-\tau$ & $462^{\mathrm{b}}$ \\
\hline M37160 & 613 & 613 & $3-596$ & $\mathrm{~F} 1-29$ & 545 \\
\hline M37161 & 613 & 613 & $3-596$ & F1-29 & $175^{\mathrm{b}}$ \\
\hline M37162 & 11661 & NA & $3-596$ & $-{ }^{\top}$ & $207^{\mathrm{b}}$ \\
\hline M37163 & 613 & 613 & $3-596$ & F1-112 & $523^{b}$ \\
\hline M37164 & 11662 & 624 & $3-596$ & $-\tau^{\top}$ & $268^{\mathrm{b}}$ \\
\hline M37166 & 613 & 613 & $3-596$ & F1-29 & 633 \\
\hline M37167 & 11663 & NA & $3-596$ & F2-7 & $548^{\mathrm{b}}$ \\
\hline M37168 & 11664 & NA & $3-596$ & F1-22 & 470 \\
\hline M37169 & 11665 & 1494 & $3-599$ & F1-105 & 262 \\
\hline M37170 & 613 & 613 & $3-596$ & F5-92 & $305^{\mathrm{b}}$ \\
\hline M37171 & 613 & 613 & $3-596$ & F1-29 & $175^{\mathrm{b}}$ \\
\hline M37172 & 11667 & 1494 & $3-596$ & F5-18 & 262 \\
\hline M37173 & 613 & 613 & $3-596$ & F1-29 & $175^{\mathrm{b}}$ \\
\hline M37174 & 11668 & NA & $3-596$ & F2-4 & 545 \\
\hline M37175 & 11669 & NA & $3-596$ & $-\tau$ & $462^{\mathrm{b}}$ \\
\hline M37176 & 11670 & NA & $3-599$ & F1-105 & 262 \\
\hline M37177 & 11671 & NA & $3-599$ & F6-3 & $308^{\mathrm{b}}$ \\
\hline M37178 & 613 & 613 & $3-596$ & F1-29 & 262 \\
\hline M37179 & 11672 & 1494 & $3-599$ & F1-105 & 262 \\
\hline M37957 & 11654 & NA & $3-596$ & F1-29 & 88 \\
\hline
\end{tabular}

$\mathrm{ST}=$ sequence type; $\mathrm{Por} \mathrm{B}=$ porin $\mathrm{B} ; \mathrm{Fet} \mathrm{A}=$ iron-regulated enterobactin; $\mathrm{NhbA}=$ neisserial heparin binding antigen. The new STs that were described for the first time in this study are indicated in bold.

a New FetA variant.

b New NhbA variant.

" fetA gene deleted.

* Isolate with $p d h C$ housekeep gene absent, no sequence type and no clonal complex assigned.

Although relatively rare, cc613 and cc1494 were predominantly associated with F1-29 (8/13; 61.5\%) and F1-105 (4/5; 80\%), respectively (Fig. 3). Furthermore, three isolates assigned to cc624 lacked the fetA gene (Table 1, Fig. 4).

The analysis of the molecular data revealed 20 different variants of intact $n h b a$, including eight new variants. The most common was variant $88(12 / 50 ; 24 \%)$ followed by $262(6 / 50 ; 12 \%)$ (Table 1$)$. When compared with the clonal complexes, the data showed that all the isolates of the variant 88 lacked association within the cc available in the PubMLST database, while most of the variant 262 (4/6; 66.7\%) were associated with the cc1494 (Table 1, Fig. 4). None of the isolates were found to contain nadA or $f H b p$ alleles.

\section{Discussion}

$N$. lactamica carriage is normally higher during the early childhood years, decreasing with the age (Cartwright et al., 1987). The participants screened in the present study were aged 11-19 years old, younger children were not included in the study and this may explain the lack of association between age and $N$. lactamica carriage prevalence. However, our data revealed a trend towards higher carriage prevalence among the youngest (11-year-old) individuals, although this was not statistically significant. Similar findings were observed with gender; a higher proportion of $N$. lactamica prevalence is usually observed among females, possibly due to prolonged or closer contact with children (Cartwright et al., 1987). However, we found no significant differences in isolation of $N$. lactamica from male or female participants. This may be due to the age of the females enrolled in this study, many of whom may have no regular or prolonged contact with young children. The overall carriage rate (4.5\%) among study participants is consistent with previous reports involving participants with higher age (Kremastinou et al., 2003; Leimkugel et al., 2007) and lower than those where young children were included (Saez-Nieto et al., 1985; Kremastinou et al., 1999; Bennett et al., 2005; Kristiansen et al., 2012).

The hypothesis that $N$. lactamica carriage could protect against meningococcal infections, either by occupying a biological niche that would otherwise be available to meningococci or by inducing natural immunity, highlights the importance of studying the population structure of N. lactamica (Lucidarme et al., 2013; Troncoso et al., 2002). Furthermore, administration of the $4 \mathrm{CMenB}$ vaccine and the presence of $N$. lactamica could be synergistic, by priming or boosting the immune response. However, vaccination could result in the elimination or prevention of $N$. lactamica carriage resulting in loss of natural immunity (Lucidarme et al., 2013). Previous studies demonstrated that N. lactamica populations are highly diverse (Bennett et al., 2005), and these findings have implications for the design of vaccines based on this organism. In the present study, the molecular data obtained by WGS was similar to those of previous studies; there was a high level of genetic variability among the $N$. lactamica isolates (Alber et al., 2001; Bennett et al., 2005; Kristiansen et al., 2012). Despite this diversity, we observed a high prevalence of cc613, followed by cc1494 and cc624, results that are consistent with those reported in the United Kingdom (Bennett et al., 2005) and Burkina Faso (Kristiansen et al., 2012).

Few studies have evaluated the distribution of outer membrane proteins among $N$. lactamica isolates (Bennett et al., 2008; Bennett et al., 2009; Lucidarme et al., 2013). In respect to the components of the $4 \mathrm{CMenB}$ vaccine, we found that none of the $N$. lactamica isolates contained NadA or FHPB, which are used in the vaccine preparation, in agreement with a previous report (Lucidarme et al., 2013). Rather, the FetA and nhba variants identified in this study were highly diverse within the same group and different STs, and only two porB variants were detected. Accordingly, as seen with the United Kingdom cc624 N. lactamica isolates (Bennett et al., 2009; Lucidarme et al., 2013), we observed fetA deletions only in isolates of cc624 and several STs for which no cc is assigned (Figs. 3 and 4). Comprehensive molecular epidemiology and surveillance including a higher number of isolates is needed to evaluate the impact of $4 \mathrm{CMenB}$ on $N$. lactamica carriage.

The analysis of the genetic diversity of the $N$. lactamica isolates using WGS provided crucial information regarding the genetic diversity of this poorly investigated bacterium. Moreover, these isolates provided valuable unexplored genomic data for further analysis that will assist our understanding of the carriage dynamics of Neisseria species.

In summary, $N$. lactamica carriage in Salvador showed no variation across the 11-19 age groups, in comparison with that seen for carriage of $N$. meningitidis. The genetic distribution and diversity of corresponding antigen genes among the $N$. lactamica isolates were similar to those reported in other studies. Continuous study on the 


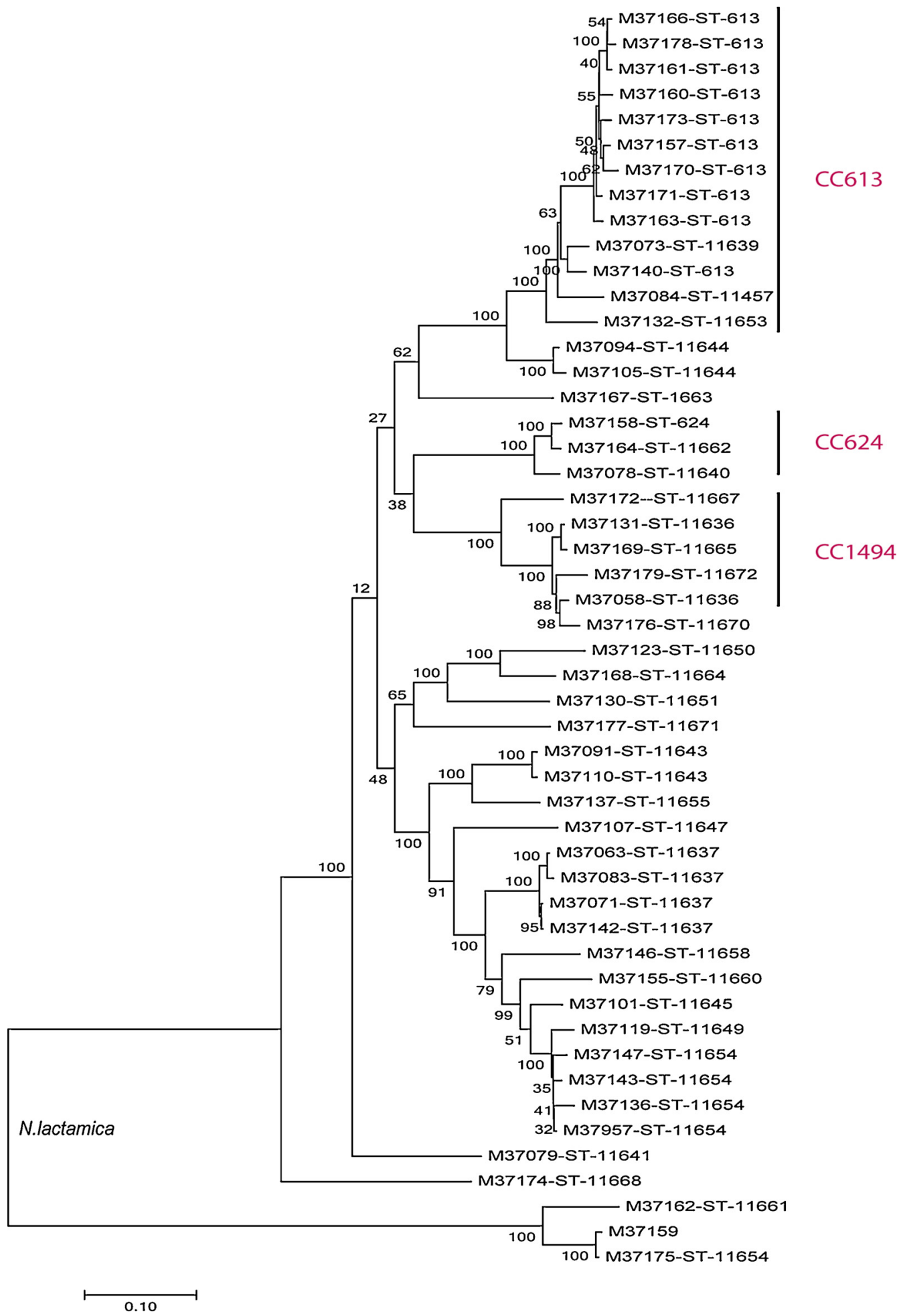

Fig. 2. Phylogenetic tree of the $N$. lactamica isolates based on the whole-genome sequence. The $N$. lactamica isolates are labelled with sample ID, sequence type (ST) and clonal complex (cc) (when assigned) they belong to. Internal nodes are labeled with bootstrap values. The scale bar is based on the 11329 positions in the core SNP matrix and indicated nucleotide substitutions per site.

characterization of circulating strains of $N$. lactamica may contribute to a better understanding of meningococcal colonization, virulence factors and vaccine responses.

\section{Acknowledgements}

This work was supported by Ministério da Saúde, Brazil (grant number TC335/2013), and Fundação de Amparo à Pesquisa do Estado da Bahia (grant number SUS 007/2014). The findings and conclusions in this report are those of the authors and do not necessarily represent the official position of the Centers for Disease Control and Prevention. 


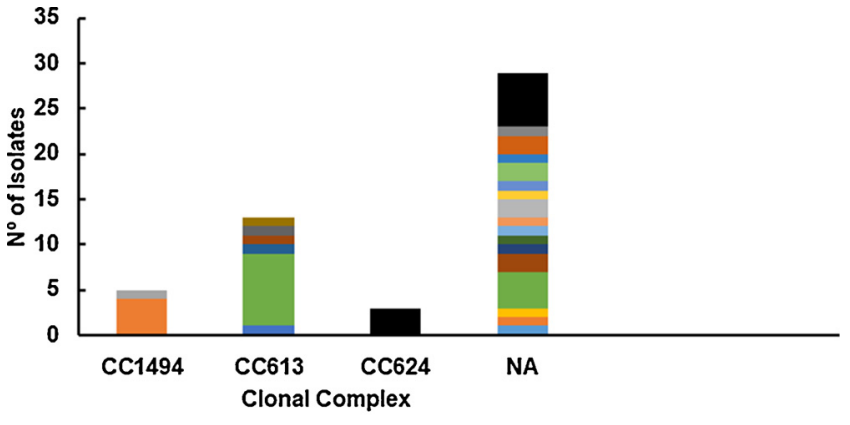

\section{$=F 1-204=F 1-105=F 5-18 \quad=F 1-22 \quad \equiv F 1-112=F 1-29 \quad=F 1-33 \quad=F 2-7$

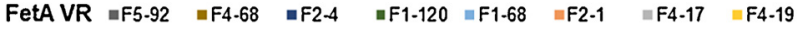 \\ $=F 5-13=F 5-88 \quad \square F 6-3 \quad=F 1-143=F 5-120$ mDeleted}

Fig. 3. FetA variants distribution among $N$. lactamica clonal complexes. VRvariants; cc- Clonal Complex; NA- clonal complex not assigned; Deleted - fetA gene deleted.

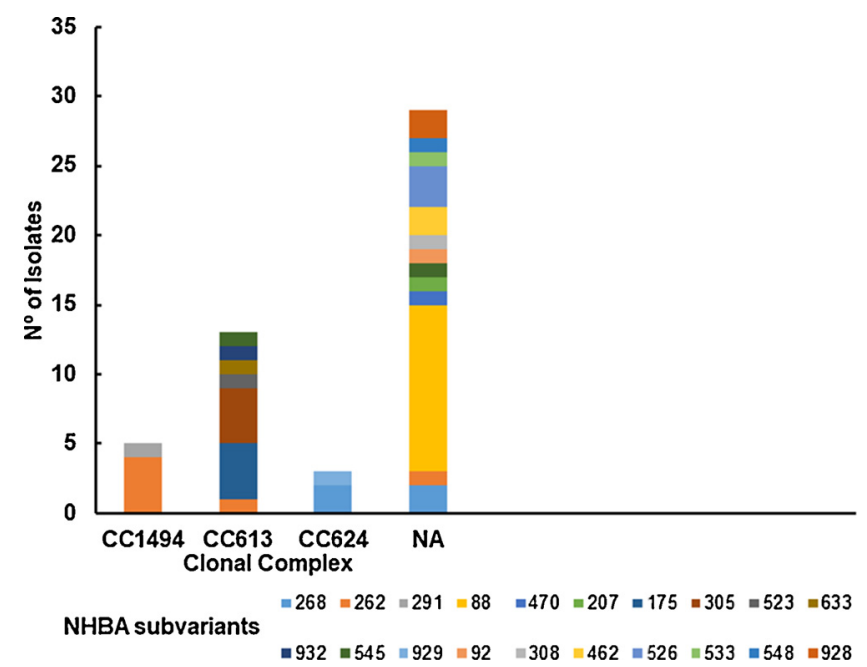

Fig. 4. NhbA variants distribution among $N$. lactamica clonal complexes. ccclonal complex; NA- clonal complex not assigned.

\section{Appendix A. Supplementary data}

Supplementary data associated with this article can be found, in the online version, at https://doi.org/10.1016/j.ijmm.2018.03.007.

\section{References}

Alber, D., Oberkotter, M., Suerbaum, S., Claus, H., Frosch, M., Vogel, U., 2001. Genetic diversity of Neisseria lactamica strains from epidemiologically defined carriers. J. Clin. Microbiol. 39, 1710-1715.

Bennett, J.S., Griffiths, D.T., McCarthy, N.D., Sleeman, K.L., Jolley, K.A., Crook, D.W., Maiden, M.C., 2005. Genetic diversity and carriage dynamics of Neisseria lactamica in infants. Infect. Immunol. 73, 2424-2432.

Bennett, J.S., Callaghan, M.J., Derrick, J.P., Maiden, M.C., 2008. Variation in the Neisseria lactamica porin, and its relationship to meningococcal PorB. Microbiology 154, 1525-1534.

Bennett, J.S., Thompson, E.A., Kriz, P., Jolley, K.A., Maiden, M.C., 2009. A common gene pool for the Neisseria FetA antigen. Int. J. Med. Microbiol. 299, 133-139.

Cann, K.J., Rogers, T.R., 1989. Detection of antibodies to common antigens of pathogenic and commensal Neisseria species. J. Med. Microbiol. 30, 23-31.

Cartwright, K.A., Stuart, J.M., Jones, D.M., Noah, N.D., 1987. The Stonehouse survey: nasopharyngeal carriage of meningococci and Neisseria lactamica. Epidemiol. Infect. 99, 591-601.

Changal, K.H., Raina, A., Altaf, S.S., 2016. Neisseria lactamica causing a lung cavity and skin rash in a renal transplant patient: first report from India. Case Rep. Infect. Dis. 1932963.

Everts, R.J., Speers, D., George, S.T., Ansell, B.J., Karunajeewa, H., Ramos, R.D., 2010.
Neisseria lactamica arthritis and septicemia complicating myeloma. J. Clin. Microbiol. $48,2318$.

Finney, M., Vaughan, T., Taylor, S., Hudson, M.J., Pratt, C., Wheeler, J.X., Vipond, C., Feavers, I., Jones, C., Findlow, J., Borrow, R., Gorringe, A., 2008. Characterization of the key antigenic components and pre-clinical immune responses to a meningococcal disease vaccine based on Neisseria lactamica outer membrane vesicles. Hum. Vaccin. 4, 23-30.

Gardner, S.N., Hall, B.G., 2013. When whole-genome alignments just won't work: kSNP v2 software for alignment-free SNP discovery and phylogenetics of hundreds of microbial genomes. PLoS One 8, e81760.

Gold, R., Goldschneider, I., Lepow, M.L., Draper, T.F., Randolph, M., 1978. Carriage of Neisseria meningitidis and Neisseria lactamica in infants and children. J. Infect. Dis. 137, 112-121.

Gorringe, A.R., Taylor, S., Brookes, C., Matheson, M., Finney, M., Kerr, M., Hudson, M., Findlow, J., Borrow, R., Andrews, N., Kafatos, G., Evans, C.M., Read, R.C., 2009. Phase I safety and immunogenicity study of a candidate meningococcal disease vaccine based on Neisseria lactamica outer membrane vesicles. Clin. Vaccine Immunol. 16, 1113-1120.

Griffiss, J.M., Yamasaki, R., Estabrook, M., Kim, J.J., 1991. Meningococcal molecular mimicry and the search for an ideal vaccine. Trans. R. Soc. Trop. Med. Hyg. 85 (Suppl. 1), 32-36.

Kim, J.J., Mandrell, R.E., Griffiss, J.M., 1989. Neisseria lactamica and Neisseria meningitidis share lipooligosaccharide epitopes but lack common capsular and class 1, 2, and 3 protein epitopes. Infect. Immun. 57, 602-608.

Kremastinou, J., Tzanakaki, G., Velonakis, E., Voyiatzi, A., Nickolaou, A., Elton, R.A., Weir, D., Blackwell, C., 1999. Carriage of Neisseria meningitidis and Neisseria lactamica among ethnic Greek school children from Russian immigrant families in Athens. FEMS Immunol. Med. Microbiol. 23, 13-20.

Kremastinou, J., Tzanakaki, G., Levidiotou, S., Markou, F., Themeli, E., Voyiatzi, A., Psoma, E., Theodoridou, M., Blackwell, C.C., 2003. Carriage of Neisseria meningitidis and Neisseria lactamica in northern Greece. FEMS Immunol. Med. Microbiol. 39, 23-29.

Kretz, C.B., Retchless, A.C., Sidikou, F., Issaka, B., Ousmane, S., Schwartz, S., Tate, A.H., Pana, A., Njanpop-Lafourcade, B.M., Nzeyimana, I., Nse, R.O., Deghmane, A.E. Hong, E., Brynildsrud, O.B., Novak, R.T., Meyer, S.A., Oukem-Boyer, O.O., Ronveaux, O., Caugant, D.A., Taha, M.K., Wang, X., Niger Response, T., 2016. Whole-genome characterization of epidemic neisseria meningitidis serogroup $\mathrm{C}$ and resurgence of serogroup W, Niger, 2015. Emerg. Infect. Dis. 22, 1762-1768.

Kristiansen, P.A., Diomande, F., Ouedraogo, R., Sanou, I., Sangare, L., Ouedraogo, A.S., Ba, A.K., Kandolo, D., Dolan Thomas, J., Clark, T.A., Preziosi, M.P., Laforce, F.M., Caugant, D.A., 2012. Carriage of Neisseria lactamica in 1- to 29-year-old people in Burkina Faso: epidemiology and molecular characterization. J. Clin. Microbiol. 50, 4020-4027.

Leimkugel, J., Hodgson, A., Forgor, A.A., Pfluger, V., Dangy, J.P., Smith, T., Achtman, M., Gagneux, S., Pluschke, G., 2007. Clonal waves of Neisseria colonisation and disease in the African meningitis belt: eight- year longitudinal study in northern Ghana. PLoS Med. 4, e101.

Lucidarme, J., Gilchrist, S., Newbold, L.S., Gray, S.J., Kaczmarski, E.B., Richardson, L., Bennett, J.S., Maiden, M.C., Findlow, J., Borrow, R., 2013. Genetic distribution of noncapsular meningococcal group B vaccine antigens in Neisseria lactamica. Clin. Vaccine Immunol. 20, 1360-1369.

Moura, A.R.S.S., Kretz, C.B., Ferreira, I.E., Nunes, A.M.P.B., De Moraes, J.C., Reis, M.G., McBride, A.J.A., Wang, X., Campos, L.C., 2017. Molecular characterization of Neisseria meningitidis isolates recovered from 11 to 19-year-old meningococcal carriers in Salvador in Salvador. PLoS One 12, e0185038.

Nunes, A.M., Ribeiro, G.S., Ferreira, I.E., Moura, A.R., Felzemburgh, R.D., de Lemos, A.P., Reis, M.G., de Moraes, J.C., Campos, L.C., 2016. Meningococcal carriage among adolescents after mass meningococcal $\mathrm{C}$ conjugate vaccination campaigns in Salvador, Brazil. PLoS One 11, e0166475.

O’Brien, K.L., Bronsdon, M.A., Dagan, R., Yagupsky, P., Janco, J., Elliott, J., Whitney, C.G., Yang, Y.H., Robinson, L.G., Schwartz, B., Carlone, G.M., 2001. Evaluation of a medium (STGG) for transport and optimal recovery of Streptococcus pneumoniae from nasopharyngeal secretions collected during field studies. J. Clin. Microbiol. 39, 1021-1024.

Saez-Nieto, J.A., Dominguez, J.R., Monton, J.L., Cristobal, P., Fenoll, A., Vazquez, J., Casal, J., Taracena, B., 1985. Carriage of Neisseria meningitidis and Neisseria lactamica in a school population during an epidemic period in Spain. J. Hyg. (Lond.) 94, 279-288.

Serruto, D., Bottomley, M.J., Ram, S., Giuliani, M.M., Rappuoli, R., 2012. The new multicomponent vaccine against meningococcal serogroup B, 4CMenB: immunological, functional and structural characterization of the antigens. Vaccine 30 (Suppl. 2), B87-97.

Tamura, K., Stecher, G., Peterson, D., Filipski, A., Kumar, S., 2013. MEGA6: molecular evolutionary genetics analysis version 6.0. Mol. Biol. Evol. 30, 2725-2729.

Toneatto, D., Pizza, M., Masignani, V., Rappuoli, R., 2017. Emerging experience with meningococcal serogroup B protein vaccines. Exp. Rev. Vaccines 16, 433-451.

Troncoso, G., Sanchez, S., Criado, M.T., Ferreiros, C.M., 2002. Analysis of Neisseria lactamica antigens putatively implicated in acquisition of natural immunity to Neisseria meningitidis. FEMS Immunol. Med. Microbiol. 34, 9-15.

Ward, M.J., Lambden, P.R., Heckels, J.E., 1992. Sequence analysis and relationships between meningococcal class 3 serotype proteins and other porins from pathogenic and non-pathogenic Neisseria species. FEMS Microbiol. Lett. 73, 283-289. 